sels. The second generation of machines now under construction (see Table II), ASDEX in Garching and TEXTOR in Jülich are directed towards research into particular aspects of Tokamak behaviour whereas the first generation machines were more general Tokamak physics research tools. ASDEX will concentrate on plasma purity and TEXTOR on plasma-wall interaction.

While most of the European programme is concentrating on Tokamak development, there is still on-going research into stellarators of which two may be mentioned: CLEO at Culham where, in collaboration with Padua the reversed field pinch is being studied too, and Wendelstein VII at Garching, currently the largest stellerator in the world.

In the USSR, it seems likely that the very large Tokamak T-20 will not now go ahead, as recent progress and other projects have diminished its usefulness. Instead, the USSR is promoting through the IAEA in Vienna the construction of a world machine following the initiative of Sigvard Eklund, the IAEA's Director-General. Japan is expressing considerable interest and it would seem that so far western Europe has shown the least enthusiasm, possibly because of exhaustion from the long drawn out negotiations on JET. A world project is, nevertheless not just empty talk
Table III. Characteristics of European Machines.

\begin{tabular}{llllrr}
\hline Machine & Location & $R, \mathrm{~cm}$ & $a, \mathrm{~cm}$ & $B, T$ & I, kA \\
T-3 & USSR & 100 & 15 & 3.5 & 120 \\
Pulsator & FRG & 70 & 12 & 2.8 & 95 \\
TFR & F & 100 & 20 & 6.0 & 400 \\
FT & I & 83 & 21 & 10.0 & 1000 \\
DITE & UK & 117 & 27 & 2.8 & 250 \\
ASDEX & FRG & 164 & 40 & 2.8 & 500 \\
TEXTOR & FRG & 175 & 50 & 2.0 & 500 \\
JET & UK & 296 & $125 / 210^{*}$ & $2.8-2.5$ & $3800-4800$ \\
\hline
\end{tabular}

$R=$ major radius of torus $:, a=$ minor radius

$B=$ toroidal field $; I=$ plasma current

* D-shaped ring

and studies are beginning on what should be built. The USSR has a vigorous programme of work on Tokamaks, including about ten at Novosibirsk doing physics, and the abandoning of T-20 signifies no lack of interest in the system.

\section{Technology}

Once the basic physics problems of a fusion reactor have been solved there will still be major areas of technology that need to be tackled. Satisfying the Lawson criterion is just the beginning. Of particular difficulty is the choice of structural materials for plasma boundary blanket and shielding, which are subjected to a massive fast neutron fluence. Another area is the question of tritium management in a circuit where the inventory might be of the order of $1 \mathrm{~kg} / \mathrm{GW}$ (th). Again the problem of producing the big magnetic fields economically, imposes superconducting technology which is still in its infancy.

Work is only just beginning on a collaborative basis in these areas and there is obviously much scope for further development. Agreement has been reached with the USA for joint work on materials, using an American high flux neutron source, and implementing agreements have been concluded with the USA and Japan for cooperation on superconductors.

\section{When}

Multi mega dollar question to $\mathrm{Pa}$ lumbo was when we might expect to see thermonuclear power becoming competitive. Refusing to be drawn so far, he was nevertheless prepared to guess that we might have a demonstration reactor working at the beginning of the next century.

\title{
Some Large Projects in European Astronomy
}

Extracts from the paper presented by L. Woltjer, Director-General of the European Southern Observatory, a collaboration of the following countries: B, DK, F, FRG, NL, S.

\section{Optical Telescopes}

Progress in optical astronomy over the past sixty years has not come about by building ever larger telescopes but in particular by improving the detector efficiency. In 1920, already one telescope of $2.5 \mathrm{~m}$ aperture was in operation and in Table I it can be seen that the majority of those telescopes completed recently and planned for the immediate future have apertures that are not very much larger. Recent telescopes in the USA have followed the same pattern; the Kitt Peak and Cerro Tololo instruments each have an aperture of $3.8 \mathrm{~m}$. Now that detectors have reached a photon collection efficiency of around $50 \%$, further improvement where the limitation is imposed by the faintness of the photon flux, can only come from larger detection areas. Angular resolution may be obtained in various ways, especially through

Table 1. European optical telescopes of recent years.

\begin{tabular}{lllll}
\hline Observatory & Location & Country/Org. & Aperture, m & Completion Date \\
Siding Spring & Aus. & UK-Aus. & 3.9 & 1974 \\
La Silla & Chile & ESO & 3.6 & 1976 \\
Zelenchukskaya & USSR & USSR & 6.0 & 1977 \\
\multirow{2}{*}{ Calar Alto } & Hawaii & Can.-F-Haw. & 3.6 & 1979 \\
& Spain & FRG & 3.5 & 1982 \\
\multirow{2}{*}{ (Infrared } & Canaries & UK & 4.2 & \\
& & Italy & 3.5 & \multirow{2}{*}{ 1978) } \\
\hline
\end{tabular}

interferometry and for the very faintest objects where detection is limited by the background, it is necessary to go out into space.

Because of the brightness of the night sky, which on a lonely mountain top still amounts to $1 / 4$ of a 20th magnitude stars / $(\operatorname{arc} s)^{2}$, stars much fainter than the 25th magnitude are essentially out of reach of terrestrial telescopes. Minimum size of a pointlike object due to atmosphere disturbance it should be noted is 1-2 arc s. Hence the need for the Space Telescope which is a joint project of NASA $(85 \%)$ and ESA $(15 \%)$. The ST is a $2.4 \mathrm{~m}$ aperture telescope with optics close to the diffraction limit, giving a point stellar image of 0.15 arc s. A further gain comes from the background, lower by one magnitude (factor $2^{1 / 2}$ ) than at the best terrestrial observatory. With such an instrument, point-like objects down to the 29th magnitude can be detected. Moreover, its ultraviolet capabilities and high angular resolution are of great importance.

Where however the limit is not background, but the smallness of the number of photons, as for example in 
Table II. Cost data for VLT and ST compared with the ESO $3.6 \mathrm{~m}$.

Investment, M SwFr

Capital charge, M SwFr/a

Operation, M SwFr/a

Annual Cost, M SwFr

Observing time, s/a

Rel. observing cost, SwFr/s

Rel. cost/photon

$\begin{array}{ccc}\text { ESO, 3.6 m } & \text { VLT, } 16 \mathbf{~ m} & \text { ST, } 2.4 \mathbf{~ m} \\ 70 & 200 & 1000 \\ 5 & 13 & 70 \\ 20 & 30 & 80 \\ 25 & 43 & 150 \\ 4 \times 10^{6} & 4 \times 10^{6} & 9 \times 10^{7} \\ 6 & 11 & 17 \\ 10 & 1 & 25\end{array}$

spectroscopic studies designed to give reasonable spectral resolution for objects in the magnitude range $18-23$, as well as in the study of objects with rapid time variations, a bigger collecting area is essential. Moreover, if extended objects like galaxies with angular sizes in excess of 1 arc $s$ are under investigation, much of the advantage of the ST is lost (unless angular resolution is needed). These then are the essential arguments for the projects now being studied: in Europe for a terrestrial $16 \mathrm{~m}$ telescope - the Very Large Telescope (or VLT) and in the US for a $25 \mathrm{~m}$ telescope - the Next Generation Telescope (or NGT).

Observations that would be made with the VLT include: 1) Redshifts of faint distant galaxies; comparatively small differences in apparent magnitude for the same redshift correspond to very different models of the Universe. 2) Sharp absorption lines in the spectra of distant quasars which may be due to matter in intergalactic space between the quasar and us; of value in evaluating models of the Universe and also galaxy formation. 3) Polarization structure of the light emitted from pulsars which is essential for ascertaining the conditions in the very strong magnetic fields around the neutron star; various $X$-ray sources show millisecond time scale structure in their light emission. 4) Composition of stars and regions of ionized gas in nearby galaxies; current information in this area is limited to our own galaxy as well as of globular clusters in other galaxies; the distribution of these objects; spectropolarimetric studies of quasars and stars...

Construction of a $16 \mathrm{~m}$ telescope poses quite new problems, as it is clear the philosophy of building a single massive light collector that has applied up to now must be abandoned. Two approaches are open: an array of smaller telescopes, e.g. four $8 \mathrm{~m}$ or sixteen $4 \mathrm{~m}$ instruments; a single instrument with a light composite mirror, the profile of which is continuously and dynamically controlled. With the multi-instrument approach, each telescope can be equip- ped with its own detection equipment. The array is then flexible in operation but expensive and limited to incoherent measurements. Alternatively it can be equipped with an optical system for light combination that allows coherent measurements but reduces materially the efficiency of photon collection because of mirror losses. Some compromise between the two is also possible. ESO, with its background of a computerized pointing and data collection system on the $3.6 \mathrm{~m}$ telescope at La Silla, is concentrating for the present on the single dish approach which it believes could be realized by 1990 .

Cost of such a large instrument is for the moment necessarily speculative, but realistic extrapolations from current experience suggest that an array could be built for about $250 \mathrm{M}$ SwFr and that this is not the most economic approach. Capital cost is not, however, the sole criterion upon which a judgment should be based, and ESO has computed the relative costs of the present $3.6 \mathrm{~m}$ as against the VLT and ST in terms also of cost per unit observing time and cost per photon detected. Calculations are based on observations of a 20th magnitude star. From Table II it can be seen that although the ST is expensive in capital investment, it has a on detachment to IRAM from CERN, Geneva.
Millimetre-wave astronomy is a relatively new branch of astronomy as the first good quality millimetre receiver was set in operation only in 1969. Since that time, the field has yielded an impressive blossom of results in spite of the still modest size of the instruments: most of the 50 interstellar molecules known today have been discovered through observations of several hundred lines in this wavelength range, and also many isotopic substitutions. These molecules, which much greater utilization factor. In the last line the efficiency of the VLT in cost per photon is evident.

\section{European VLBI Network}

Plans are now advanced for having a European Very Long Baseline Interferometer network. Essentially international, the project is ideally suited to a European cooperation where each country has its own observing facilities but the results come from a combination of all.

At present, data from existing stations are recorded separately on tapes with a caesium clock providing the time standard, but the process of combination is slow and tedious. Proposals have therefore ben made to ESA for a dedicated communications satellite which would allow the European VLBI to operate in real time. Moreover, it would lead to great progress in astrometry in particular in connexion with the problem of establishing a fundamental reference frame, and in geophysics by making possible the detection of crustal motions of the order of $1 \mathrm{~cm}$ over $1000 \mathrm{~km}$.

\section{Other Collaborative Projects}

In the European space programme there is under construction a large satellite for X-ray studies - Exosal and an infrared satellite - IRAS due for completion in 1980/81 that will, for the first time, make a full inventory of the infrared sky down to faint flux levels. Plans for other possible satellites include an astrometric satellite and an XUV satellite which would search for sources in the hard ultraviolet and soft X-ray range - a region where at present very little is known.

\section{Radio Astronomy Projects in the Millimetre Range}

A summary of his talk on the Franco-German project IRAM, prepared by Kjell Johnsen,

make up at least half the mass of the interstellar gas in the Galaxy, are mainly found in previously unknown massive and relatively dense molecular clouds which appear to be the principal sites of star formation. Radio line and continuum observations and radio observations of interstellar masers, as well as observations in the infrared and submillimetre range, have allowed preliminary mapping of some of these clouds and have revealed dense and hot spots which 